\title{
MicroRNA function is required for neurite outgrowth of mature neurons in the mouse postnatal cerebral cortex
}

\author{
Janet Hong ${ }^{1}$, Haijun Zhang ${ }^{1}$, Yoko Kawase-Koga ${ }^{1,2}$ and Tao Sun ${ }^{1}$ * \\ ' Department of Cell and Developmental Biology, Cornell University Weill Medical College, New York, NY, USA \\ 2 Department of Oral and Maxillofacial Surgery, The University of Tokyo Hospital, Tokyo, Japan
}

\author{
Edited by: \\ Tommaso Pizzorusso, Istituto \\ di Neuroscienze, Consiglio Nazionale \\ delle Ricerche, Italy \\ Reviewed by: \\ Davide De Pietri Tonelli, Fondazione \\ Istituto Italiano di Tecnologia, Italy \\ Jie Zhang, University of Texas Health \\ Science Center at San Antonio, USA \\ *Correspondence: \\ Tao Sun, Department of Cell and \\ Developmental Biology, Cornell \\ University Weill Medical College, \\ 1300 York Avenue, Box 60, New York, \\ NY 10065, USA \\ e-mail: tas2009@med.cornell.edu
}

\begin{abstract}
The structure of the postnatal mammalian cerebral cortex is an assembly of numerous mature neurons that exhibit proper neurite outgrowth and axonal and dendritic morphology. While many protein coding genes are shown to be involved in neuronal maturation, the role of microRNAs (miRNAs) in this process is also becoming evident. We here report that blocking miRNA biogenesis in differentiated neurons results in microcephaly like phenotypes in the postnatal mouse brain. The smaller brain defect is not caused by defective neurogenesis, altered neuronal migration or significant neuronal cell death. Surprisingly, a dramatic increase in neuronal packing density within the postnatal brain is observed. Loss of miRNA function causes shorter neurite outgrowth and smaller soma size of mature neurons in vitro. Our results reveal the impact of miRNAs on normal development of neuronal morphology and brain function. Because neurite outgrowth is critical for neuroregeneration, our studies further highlight the importance of miRNAs in the treatment of neurological diseases.
\end{abstract}

\section{Keywords: miRNAs, Dicer, neurogenesis, neurite outgrowth, cerebral cortex}

\section{INTRODUCTION}

In the mammalian cerebral cortex, projection neurons are generated from radial glial cells (RGCs) and intermediate progenitors (IPs) that reside in the ventricular zone (VZ) and subventricular zone (SVZ), respectively (Noctor et al., 2001; Rakic, 2003; Haubensak et al., 2004; Englund et al., 2005; Gotz and Huttner, 2005). Postmitotic neurons (PNs) differentiate and migrate into the cortical plate $(\mathrm{CP})$, in which PNs are organized in an insideout six layered structure, with earliest born neurons in the deep layers and later born neurons in the upper layers (Guillemot, 2005; Molyneaux etal., 2007). Proper neurite outgrowth and axonal and dendritic morphogenesis are critical for neuronal maturation, synaptic formation, and neuronal function (Frank and Tsai, 2009; Merot et al., 2009). Molecular mechanisms regulating neuronal differentiation and maturation remain an exciting research topic.

The importance of microRNAs (miRNAs)-mediated neurogenesis and neuronal maturation in the central nervous system (CNS) has drawn significant attention (Kosik, 2006; Fineberg et al., 2009; Shi et al., 2010; Bian and Sun, 2011). MiRNAs are approximately 22 nucleotide (nt) endogenous non-coding small RNAs (Lee et al., 1993; Wightman et al., 1993). A mature miRNA recognizes a complementary sequence in the $3^{\prime}$-untranslated region (3'-UTR) of its target messenger RNA (mRNA) and affects mRNA stability and/or silences protein translation (Carthew and Sontheimer, 2009; Kim et al., 2009). Because miRNA precursors are processed into mature miRNAs by the RNAase III enzyme Dicer, the role of miRNAs in neurogenesis has been demonstrated by regional-specific deletion of Dicer expression in the CNS using different Cre lines (Volvert et al., 2012; Zhang et al., 2012). For example, Dicer ablated knockout (Ko) mice in PNs using the CaMKII-Cre line display impaired dendritic branching in pyramidal neurons in the CA1 region of the hippocampus (Davis et al., 2008; Hebert et al., 2010). These studies indicate the importance of miRNA functions in morphogenesis of mature neurons in the brain.

In this study, we demonstrate the critical role of miRNAs in neurite outgrowth of mature cortical neurons. Blocking miRNA biogenesis in PNs in the mouse cortex at perinatal stages does not significantly affect neurogenesis, neuronal survival, and layer organization. However, the neuronal packing density is greatly increased in the CP, resulting in a significantly reduced cortical size. Correspondingly, neurite outgrowth and soma size development are significantly reduced in cultured Dicer Ko PNs. Our results demonstrate that miRNA functions are required for proper neuronal maturation. Moreover, our studies suggest a potential role of miRNAs in promoting neurite outgrowth in the treatment of neurodegenerative diseases.

\section{MATERIALS AND METHODS GENERATION OF Dicer CONDITIONAL KNOCKOUT MICE}

The floxed Dicer transgenic mice (Dicer flox/flox; C57/BL6 $\times 129$ background; kindly provided by the Greg Hannon's lab at the Cold Spring Harbor Laboratory; Murchison et al., 2005) were bred with Nex-Cre mice (C57/BL6 background, provided by Drs M. Schwab and K. Nave at Max-Planck-Institute of Experimental Medicine, Goettingen, Germany; Goebbels et al., 2006) to generate Nex-CreDicer Ko (Nex-Cre; Dicerflox/flox $)$ animals.

For staging of embryos, mid-day of the day of vaginal plug formation is considered embryonic day 0.5 (E0.5), and the first $24 \mathrm{~h}$ after birth is defined as postnatal day 0 (P0). Animal use was overseen by the Animal Facility at the Weill Cornell Medical College. 


\section{GENOTYPING OF Dicer CONDITIONAL KNOCKOUT MICE}

Mouse tail tip biopsies were used for genotyping by polymerase chain reaction reactions using the following primer pairs: for Cre, 5'-TAAAGATATCTCACGTACTGACGGTG-3' and 5'TCTCTGACCAGAGTCATCCTTAGC-3' (product size: $350 \mathrm{bp}$ ); for Dicer, 5'-ATTGTTACCAGCGCTTAGAATTCC-3' and $5^{\prime}$ GTACGTCTACAATTGTCTATG-3' (product sizes: 767 bp from the floxed Dicer allele and 560 bp from the wild-type Dicer gene).

\section{BREEDING THE NeX-Cre LINE WITH FLOXED LacZ REPORTER MICE}

To localize the Cre activity sites, Nex-Cre transgenic mice were crossed with homozygous ROSA26 floxed LacZ reporter mice, obtained from Jackson Laboratories (Bar Harbor, Maine). The ROSA26 mice carry a loxP-flanked transcriptional "STOP” DNA sequence that prevents the transcription of the $L a c Z$ gene. Only the cells that express the Cre recombinase can remove the "STOP" sequence and subsequently activate the transcription of the LacZ gene. Cells which express LacZ produce a blue color in the $\beta$-galactosidase assay (X-gal staining).

\section{B-GALACTOSIDASE ACTIVITY ASSAY}

Mouse brains were dissected in ice-cold $1 \times$ phosphate buffered saline (PBS) and placed in 4\% paraformaldehyde (PFA) in PBS for $15 \mathrm{~min}$ at room temperature. Fixed brains were washed in PBS for $3 \times 5 \mathrm{~min}$ and sectioned coronally $(100 \mu \mathrm{m})$ using a Leica vibratome (Leica, VT1000 S). Brain sections were washed three times in a wash solution $(0.1 \mathrm{M}$ phosphate buffer and $2 \mathrm{mM} \mathrm{MgCl}_{2}$ ) and subjected to a 5-bromo-4-chloro-3-indolyl- $\beta$ D-galactopyranoside (X-gal) solution $(1 \mathrm{mg} / \mathrm{ml} \mathrm{X}$-gal and $5 \mathrm{~mm}$ potassium ferrocyanide, $5 \mathrm{~mm}$ potassium ferricyanide in wash buffer) for $30 \mathrm{~min}$ to $1 \mathrm{~h}$ at $37^{\circ} \mathrm{C}$. The reaction was quenched by washing sections three times in wash solution and incubating them in 4\% PFA in PBS for $5 \mathrm{~min}$ at room temperature. The sections were washed three times in wash solution and mounted with a coverslip. The images were collected using a Leica digital camera under a dissection scope (Leica, MZ16F).

\section{TISSUE PREPARATION AND IMMUNOHISTOCHEMISTRY}

Mouse brains were collected and fixed in $4 \%$ PFA in PBS at $4^{\circ} \mathrm{C}$ overnight, followed by incubating in $30 \%$ sucrose in PBS. Brain tissues were embedded in optimal cutting temperature (OCT) and stored at $-80^{\circ} \mathrm{C}$ until use. Brains were sectioned coronally $(14 \mu \mathrm{m})$ using a Leica cryostat (Leica, CM3050 S).

For antigen recovery, sections were incubated in heated (95$100^{\circ} \mathrm{C}$ ) antigen recovery solution $[1 \mathrm{mM}$ ethylenediaminetetraacetic acid (EDTA), $5 \mathrm{mM}$ Tris, $\mathrm{pH} \mathrm{8.0]} \mathrm{for} \mathrm{15-20} \mathrm{min,} \mathrm{and}$ cooled down for 20-30 min. Before applying antibodies, sections were blocked in 10\% normal goat serum (NGS) in PBS with $0.1 \%$ Tween-20 (PBT) for $1 \mathrm{~h}$. Sections were incubated with primary antibodies at $4^{\circ} \mathrm{C}$ overnight and visualized using goat anti-rabbit IgG-Alexa-Fluor-488 and/or goat anti-mouse IgGAlexa-Fluor-546 (1:350, Molecular Probes) for $1.5 \mathrm{~h}$ at room temperature. Images were captured using a Leica digital camera under a fluorescent microscope (Leica DMI6000B).

Primary antibodies against the following antigens were used: bromodeoxyuridine (BrdU; 1:50, DSHB), Ki67 (1:500, Abcam),
Tbr1 (1:2500, Abcam), Ctip2 (1:1000, Abcam), Cux1 (1:200, Santa Cruz), Satb2 (1:1000, Abcam), $\beta$-tubulin III (TuJ1; $1: 500$, Chemicon), Map2 (1:500, Chemicon), and NeuN (1:300, Chemicon).

\section{NISSL STAINING}

Sections $(14 \mu \mathrm{m})$ were processed through incubation in the following solutions in order: ethanol/chloroform (1:1, overnight), $100 \%$ ethanol (30 s), 95\% ethanol (30 s), distilled water (30 s, twice), cresyl violet (3-5 min), distilled water ( $2 \mathrm{~min}$, three times), $50 \%$ ethanol (2 $\mathrm{min}), 95 \%$ ethanol (5-30 $\mathrm{min}), 100 \%$ ethanol ( 5 min, twice), xylene ( 3 min, twice), and then mounted with a coverslip. The images were collected using a Leica digital camera under a dissection scope (Leica, MZ16F).

\section{IN SITU HYBRIDIZATION}

In situ hybridization for miRNA expression was performed according to previously published methods with modifications using locked nucleic acid (LNA) probes (Obernosterer et al., 2007). Briefly, after fixation with 4\% PFA, acetylation with acetylation buffer (13.33\% triethanolamince, $2.5 \%$ acetic anhydride, $20 \mathrm{mM} \mathrm{HCl})$, treatment of proteinase $\mathrm{K}(10 \mathrm{mg} / \mathrm{ml}$, IBI Scientific) and pre-hybridization [ $1 \times$ saline-sodium citrate (SSC), 50\% formamide, $0.1 \mathrm{mg} / \mathrm{ml}$ salmon sperm DNA solution, $1 \times$ Denhardt, $5 \mathrm{mM}$ EDTA, pH 7.5], brain sections were hybridized with digoxigenin (DIG)-labeled LNA probes at a proper temperature $\left(\mathrm{Tm}-22^{\circ} \mathrm{C}\right)$ overnight. After washing with pre-cooled wash buffer ( $1 \times$ SSC, $50 \%$ formamide, $0.1 \%$ Tween-20) and $1 \times$ maleic acid buffer containing Tween 20 (MABT), sections were blocked with blocking buffer $(1 \times$ MABT, 2\% blocking solution, 20\% heat-inactived sheep serum) and incubated with anti-DIG antibody $\left(1: 1,500\right.$, Roche) at $4^{\circ} \mathrm{C}$ overnight. Brain sections were washed with $1 \times$ MABT and staining buffer $(0.1 \mathrm{M}$ $\mathrm{NaCl}, 50 \mathrm{mM} \mathrm{MgCl} 2,0.1 \mathrm{M}$ Tris- $\mathrm{HCl}, \mathrm{pH}$ 9.5), stained with $\mathrm{BM}$ purple (Roche) at room temperature until ideal intensity. The microRNA LNA probes (Exiqon) were $3^{\prime}$ end labeled with DIGddUTP with terminal transferase using the DIG-3' end labeling kit (Roche).

The images of in situ hybridization were collected using a Leica digital camera under a dissection scope (Leica, MZ16F).

\section{BrdU INCORPORATION}

To assess proliferation of neural progenitors in the developing cortex, one dose of BrdU ( $50 \mu \mathrm{g} / \mathrm{g}$ body weight) was administrated by intraperitoneal injection to timed-pregnant female mice half an hour before sacrifice.

\section{TUNEL ASSAY}

To identify apoptotic cells in the cortex, we performed a TUNEL (terminal deoxynucleotidyl transferase dUTP nick end labeling) assay using an Apop Tag Fluorescein in situ Apoptosis detection kit (Chemicon) on $14-\mu \mathrm{m}$ frozen sections. This assay was performed according to the manufacturer's instructions.

\section{CELL COUNTING IN THE CORTICAL WALL}

Coronal sections were collected in the medial cortical region (at levels between the anterior commissure and the anterior hippocampus). At least four sections from each brain and three brains from different litters were chosen for antibody labeling and 
TUNEL assay. For Figures 2 and 3, positive cells were quantified in fixed areas of $186 \mu \mathrm{m} \times 1200 \mu \mathrm{m}$ in the cortical wall of P5 and P10 cortices. For Figure 4, positive cells were quantified in fixed areas of $186 \mu \mathrm{m} \times 186 \mu \mathrm{m}$ in the cortical wall of P5 and P10 cortices.

\section{PRIMARY NEURONAL CULTURES}

Neuronal cultures were performed according to established protocols (Yu et al., 2005) with modifications. Briefly, the dorsal cortex was dissected from the P0 brain, and transferred to pre-cooled Hanks' balanced salt solution (HBSS) medium. Tissue was dissociated with $0.5 \mathrm{mg} / \mathrm{ml}$ DNAse I (Sigma D4527) in HBSS for $2 \mathrm{~min}$ at $37^{\circ} \mathrm{C}$ and mechanically triturated with fire-polished Pasteur pipettes into a single cell suspension. Cortical neurons were plated onto poly-L-lysine (PLL) and Laminin treated coverslips at $5 \times 10^{4}$ cells/well in 24-well plates. Neuronal cultures were maintained in neuronal medium [Dulbecco's modified Eagle medium (DMEM)/F12, N2, B27, glucose, $\mathrm{NaHCO}_{3}$, HEPES (4-(2hydroxyethyl)-1-piperazineethanesulfonic acid)] with fibroblast growth factor 2 (FGF-2; $20 \mathrm{ng} / \mathrm{ml}$; Invitrogen) treatment for the first 24 h only. Afterward, cells were cultured in neuronal medium only and medium was changed every $2-3$ days.

Primary neurons were fixed after 10 days in vitro (DIV 10) with 4\% PFA in PBS for $30 \mathrm{~min}$ at room temperature. Before applying antibodies, cells were blocked in 10\% NGS in PBS with $0.3 \%$ Triton $\mathrm{X}-100$ for $1 \mathrm{~h}$. Cells were incubated with primary antibodies at $4^{\circ} \mathrm{C}$ overnight and visualized using goat anti-rabbit IgG-AlexaFluor-488 and/or goat anti-mouse IgG-Alexa-Fluor-546 (1:350, Molecular Probes) for $1.5 \mathrm{~h}$ at room temperature. Images were captured using a Leica digital camera under a fluorescent microscope (Leica DMI6000B).

\section{ANALYSIS OF NEURITE GROWTH AND SOMA SIZE}

Typically, pictures of 30-50 neurons from three separate coverslips from each experiment were taken using a Leica digital camera under a fluorescent microscope (Leica DMI6000B). Representative cells with strong Map2 and Tuj1 immunoreactivity labeling neurite (axonal and dendritic) processes were analyzed. Neurites that had lengths that were at least twice the diameter of the cell body were measured. Neurite lengths from the soma and soma size area were traced and measured using Image J software and the data were compiled and analyzed using the Excel program (Microsoft).

\section{STATISTIC ANALYSIS}

At least three Nex-Cre-Dicer Ko (Ko) and three control (Ctrl) animals were used for all statistical analyses. Data were shown as mean \pm SEM. Statistical comparison was made by analysis of variance (unpaired $t$-test or analyses of variance). Additional details regarding the $n$ (number of animals) or $N$ (number of neurites or cells) are found in the pertinent figure legend.

\section{RESULTS}

\section{CORTICAL GROWTH DEFECTS IN Nex-Cre-Dicer KNOCKOUT MICE}

To examine the role of miRNAs in the maturation of differentiated neurons, we conditionally ablated Dicer expression in PNs in the mouse cerebral cortex utilizing a Cre-loxp system. A floxed
Dicer mouse line (Dicerflox/flox $)$ with two loxP sites flanking exon 22 and exon 23, which encode the RNAase III domains of Dicer, were bred with a Nex-Cre mouse line to generate Nex-Cre-Dicer Ko mice (Figure 1A). The Nex-Cre line displays activity by E13.5 and is prominently expressed in differentiating neurons of the dorsal telencephalon without affecting proliferating precursor cells of the VZ (Goebbels et al., 2006). Proliferating precursor cells can be detected by labeling cells in the $S$ phase with a $30 \mathrm{~min}$ pulse of BrdU, and in the G1, S, G2, and M phase with Ki67. Indeed, quantification of $\mathrm{BrdU}^{+}$and $\mathrm{Ki}^{+}{ }^{+}$cells revealed no change in E15.5 Nex-Cre-Dicer Ko cortices compared to controls (data not shown). As such, Dicer and consequently miRNA production was conditionally ablated in PNs in the cortex after Cre recombination, as demonstrated by X-gal staining in P1 cortices of mice bred between the Nex-Cre line and the Rosa26-LacZ reporter line (Figure 1B).

Inactivation of Dicer in differentiated neurons caused markedly reduced postnatal growth. Moreover, Nex-Cre-Dicer Ko mice could not survive past P23, presumably due to starvation and dehydration after weaning. At P1, the brain size of Nex-Cre-Dicer Ko mice was comparable to that of controls (data not shown). However, gross brain morphology at $\mathrm{P} 10$ revealed a significant size reduction in Nex-Cre-Dicer Ko brains compared to controls (Figure 1C). Quantification of the body and brain weights of P10 Dicer Ko mice showed a significant reduction compared to controls, with a more profound reduction in brain weight (Figure 1D). Next, cortical morphology was analyzed in coronal sections of P10 brains by Nissl staining. While overall cortical lamination appeared normal, the thickness of the cortical wall was significantly reduced in Nex-Cre-Dicer Ko brains compared to controls (Figure 1E).

To verify that the brain phenotypes were caused by miRNA loss, we performed miRNA in situ hybridization in control and Dicer Ko brains. Three brain-enriched miRNAs, miR-9, Let-7, and miR128, were utilized. We found that expression levels of all three miRNAs were reduced in P1 cortices and almost diminished in P10 cortices, suggesting a progressive loss of miRNAs due to Dicer deletion (Figure 1F and data not shown).

Our results indicate that Dicer deletion in differentiated neurons in the developing brain causes early postnatal death, reduced body and brain weights, and severe reduction of the cortical wall.

\section{DEPLETION OF MIRNA FUNCTION IN POSTMITOTIC NEURONS DOES NOT SIGNIFICANTLY AFFECT CORTICAL LAMINATION AND NEURONAL PRODUCTION}

Due to the significant reduction in cortical wall thickness, we investigated the effects of Dicer ablation on the generation of early- and late-born neurons. During mouse cortical development, early-born neurons generated at E12.5-E13.5 migrate and form deep cortical layers VI and V, and express Tbr1 and Ctip2, respectively (Arlotta et al., 2005; Kolk et al., 2006; Chen et al., 2008; Han et al., 2011). Late-born neurons are generated at E14.5-E18.5 and migrate to form the upper neuronal layers II-IV above the deep cortical layers, and can be detected by Satb2 and Cux1 expression (Alcamo et al., 2008; Britanova et al., 2008; Cubelos et al., 2010). We first examined early- and late-born neuron production in the P5 cortex. Quantification of 
A

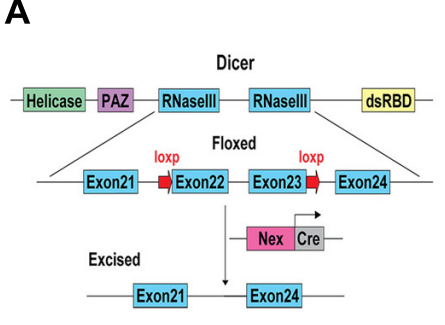

B
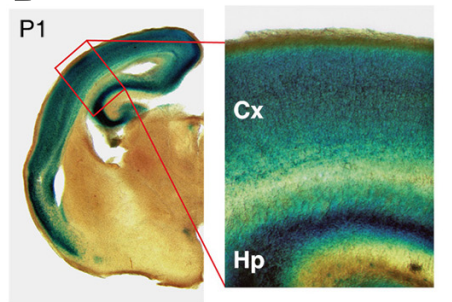

$\mathbf{F}$
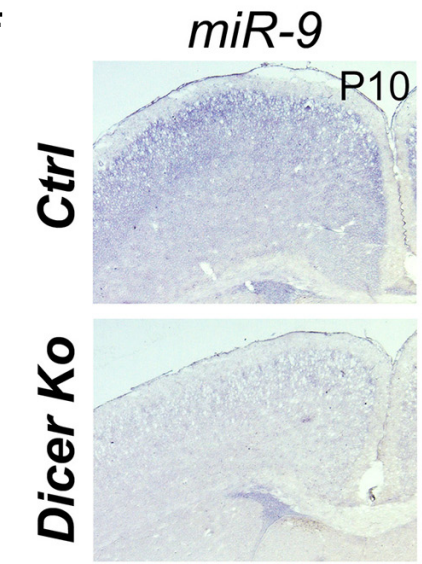

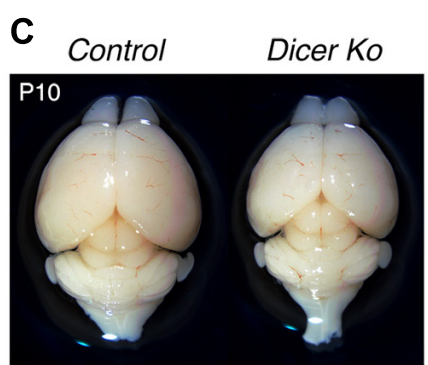

D
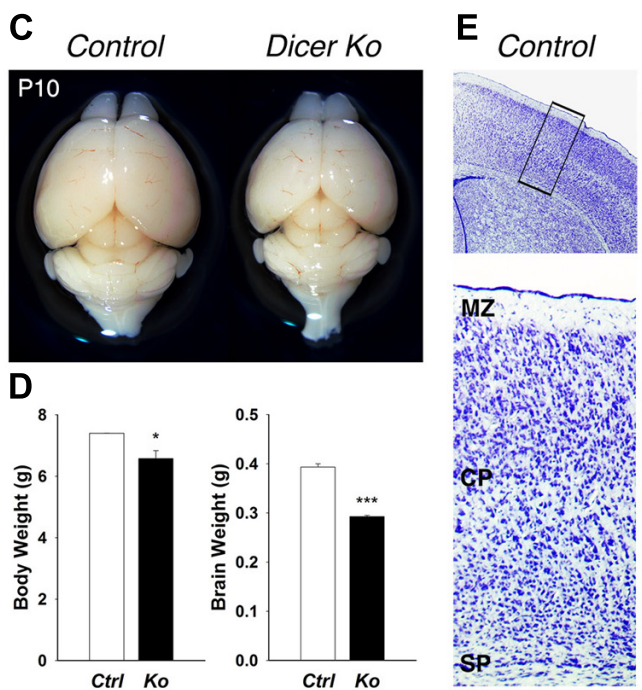

Dicer Ko
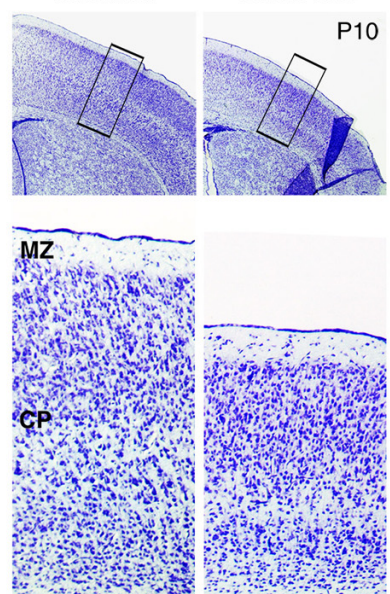

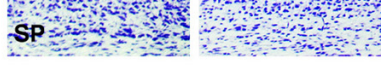

Let-7

$\operatorname{miR}-128$
FIGURE 1 | Conditional ablation of Dicer in postmitotic neurons of the cerebral cortex results in a smaller cortex and reduced thickness of the cortical wall. (A) Dicer targeting construct. The N-terminal RNA helicase domain, piwi argonaute and zwille (PAZ) domain, two ribonuclease III domains, and a double-stranded RNA- binding domain (RBD) are labeled. The exon 22 and exon 23 of Dicer are conditionally excised after Nex-Cre recombination. (B) X-gal staining at the level of the cortex in a Nex-Cre and Rosa26-LacZexpressing mouse at P1 illustrating cortical specificity of the Nex-Cre line. The red box indicates the region shown at higher magnification. The cortex $(C x)$ and hippocampus ( $\mathrm{Hp}$ ) are labeled. (C) Appearance of representative brains from P10 control and Nex-Cre-Dicer Ko mice (litter mates). (D) Body and brain weights of control (Ctrl) and Nex-Cre-Dicer Ko (Ko) mice at P10. (E) Coronal sections of P10 brains with Nissl staining of control and Nex-Cre-Dicer Ko mice. The black boxes indicate the region shown at higher magnification. The subplate (SP), cortical plate (CP), and marginal zone (MZ) are labeled. (F) In situ hybridization of miR-9, Let-7, and miR-128 in control and Dicer Ko cortices at P10. Data are presented as mean \pm SEM; $n \geq 3$ in all genotypes; $p$ values in relation to control $\left({ }^{*} p<0.05,{ }^{* *} p<0.00002\right)$. early-born neurons with $\mathrm{Tbr}^{+}{ }^{+}$and Ctip2 ${ }^{+}$cells revealed no significant difference between control and Nex-Cre-Dicer Ko cortices (Figures 2A,B). For late-born neurons, Satb2 ${ }^{+}$cells were unaffected but Cux1 ${ }^{+}$cells were slightly decreased in Dicer Ko cortices compared to controls (Figures 2A,B). Next, we analyzed neuronal production in the P5 cortex. Quantification of neuron and cell numbers by NeuN and DAPI immunostaining showed no significant difference in Nex-Cre-Dicer Ko cortices compared to controls (Figures 2C,D).

Given that Dicer ablation did not reveal a significant defect in cortical lamination and neuronal production despite the reduced cortical thickness, we investigated the possibility of neuronal cell death. Apoptotic cells in the cortex were detected by TUNEL assay. At P5, there was a significant increase in apoptotic cells in Nex-Cre-Dicer Ko cortices compared to controls, which was not detected in P1 cortices (Figures 2E,F and data not shown). Moreover, $\mathrm{TUNEL}^{+}$cells in Dicer Ko brains were localized in the far-upper cortical layer at the marginal zone boundary, suggesting apoptosis of a subset of late-born neurons (Figures $2 \mathbf{E a}^{\prime}, \mathbf{b}^{\prime}$ ).

We further examined cortical lamination, neuronal production, and apoptosis in P10 control and Nex-Cre-Dicer Ko brains. Numbers of Tbr $1^{+}$and Ctip $2^{+}$early-born neurons were increased and decreased in Dicer Ko cortices, respectively, compared to controls (Figures 3A,B). Conversely, quantification of Satb2 ${ }^{+}$ and $\mathrm{Cux}^{+}$late-born neurons revealed no significant difference between control and Dicer Ko cortices (Figures 3A,B). Subsequently, we analyzed neuron and cell number by NeuN and DAPI immunostaining in the P10 cortex. Interestingly, countings of 


\section{A Tbr1/DAPI}

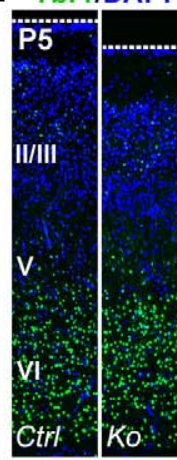

Ctip2/DAPI

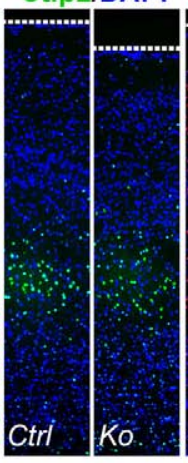

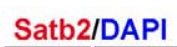

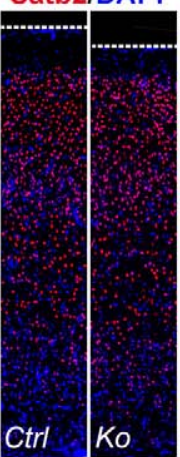

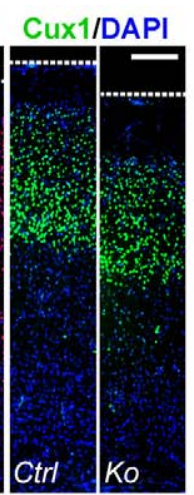

\section{B}
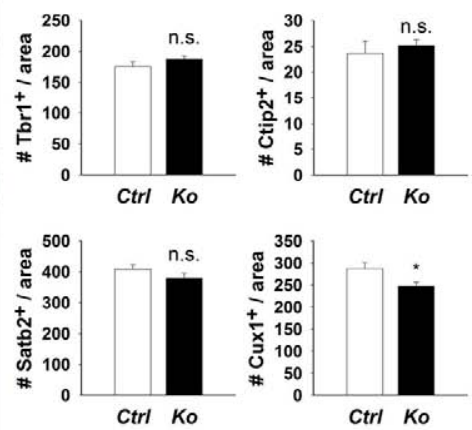

C
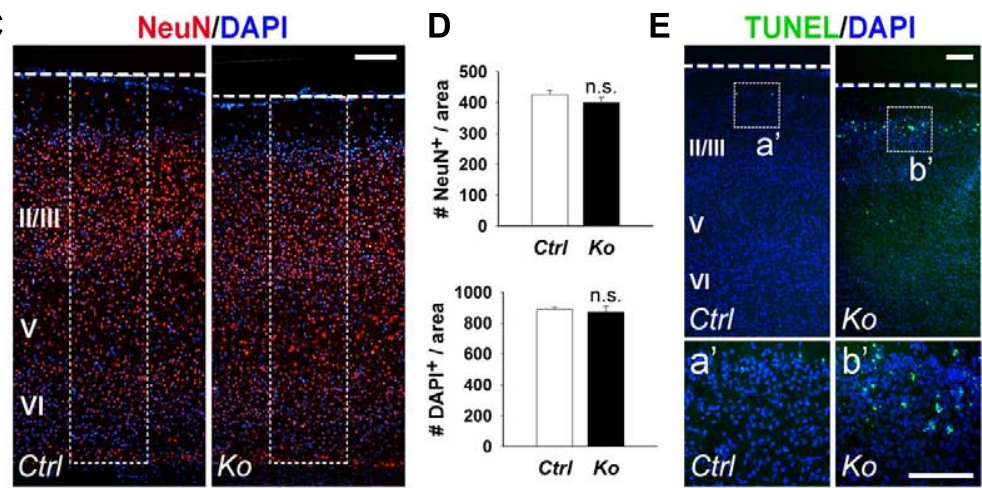

$\mathbf{F}$

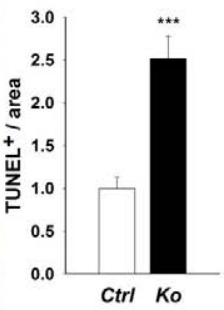

FIGURE 2 | Conditional Dicer loss in mature neurons does not significantly alter cortical lamination and neuronal production, and exhibits a transitory increase in cell death at P5. (A,B) The numbers of early-born neurons labeled with Tbr1 and Ctip2 were unaffected in P5 Nex-Cre-Dicer $K_{0}(K o)$ cortices, compared to controls (Ctrl). The numbers of late-born neurons labeled with Satb2 were unaffected and Cux1 were decreased in P5 Dicer Ko cortices, compared to controls. (C,D) Numbers of $\mathrm{NeuN}^{+}$and $\mathrm{DAPI}^{+}$cells were unaffected in the cortical wall of P5 Dicer Ko cortices compared to controls. The dashed box indicates the area of quantification. (E) TUNEL assay of coronal cryosections of P5 control and Dicer Ko cortices. The dashed box indicates the region shown at higher magnification in panel $\mathbf{a}^{\prime}, \mathbf{b}^{\prime}$. (F) Normalized quantification of TUNEL ${ }^{+}$cells per area in the cortical wall of P5 control and Dicer Ko brains. Cortical layers $(\mathrm{VI}),(\mathrm{V})$, and (II/III) are labeled. Scale bar: $100 \mu \mathrm{m}$. Data are presented as mean $\pm \mathrm{SEM} ; n \geq 3$ in all genotypes; $p$ values in relation to control $\left({ }^{*} p<0.04,{ }^{* *} p<0.0002\right)$. n.s., not significant.
$\mathrm{NeuN}^{+}$and $\mathrm{DAPI}^{+}$cells within fixed columns of the cortical wall revealed no significant alterations in neuronal or cell number in Nex-Cre-Dicer Ko cortices, despite its significantly thinner cortex (Figures 3C,D). Further TUNEL analysis in the P10 cortex revealed no significant differences in the numbers of apoptotic cells in Dicer Ko and control brains (Figures 3E,F).

Our results indicate that even though the numbers of early- and late-born neurons, and apoptotic cells show temporal changes in postnatal cortices of Nex-Cre-Dicer Ko mice, overall cortical lamination and neuronal production remain undisrupted.

\section{CONDITIONAL Dicer ABLATION AFFECTS NEURON AND CELL PACKING DENSITY WITHIN THE CORTEX}

Considering that inactivation of Dicer in PNs did not adversely affect cortical lamination and neuronal production and only had a transient effect on cell survival, we investigated the cause of the smaller cortex in Nex-Cre-Dicer Ko mice further. We analyzed the density of neurons and cells by quantifying the number of $\mathrm{NeuN}^{+}$and $\mathrm{DAPI}^{+}$cells within uniform boxed areas in the upper and lower regions of the CP. At P5, there were no alterations in $\mathrm{NeuN}^{+}$neuron and $\mathrm{DAPI}^{+}$cell density in Dicer Ko cortices compared to controls (Figures 4A,B). However, P10 Dicer Ko cortices revealed significantly increased $\mathrm{NeuN}^{+}$and $\mathrm{DAPI}^{+}$ cell numbers compared to controls, indicating increased density and packing of cells within the cortex during the stage of neuronal maturation (Figures 4C,D). These results demonstrate that Dicer ablation in PNs does not cause defective neuronal production but alters the neuronal packing density within the cortex.

\section{LOSS OF Dicer CAUSES ABNORMAL NEURONAL MATURATION WITH SHORTER NEURITE OUTGROWTH AND SMALLER CELL BODY SIZE}

Given that the packing density of neurons was dramatically increased in the Nex-Cre-Dicer Ko cortices, we decided to further analyze neuronal morphology in vitro. This was done by harvesting cortical neurons from P0 mouse brains and culturing them under differentiation conditions using previously described methods with modifications (Figure 5A; Yu et al., 2005). After 10 days in vitro (DIV 10), cultures of differentiated neurons from control and Dicer Ko cortices were labeled with antibodies against Map2 and Tuj1 to illustrate neurites. We found that Dicer Ko neurons displayed significantly shorter neurites and processes compared to controls (Figures 5B,C). We next 


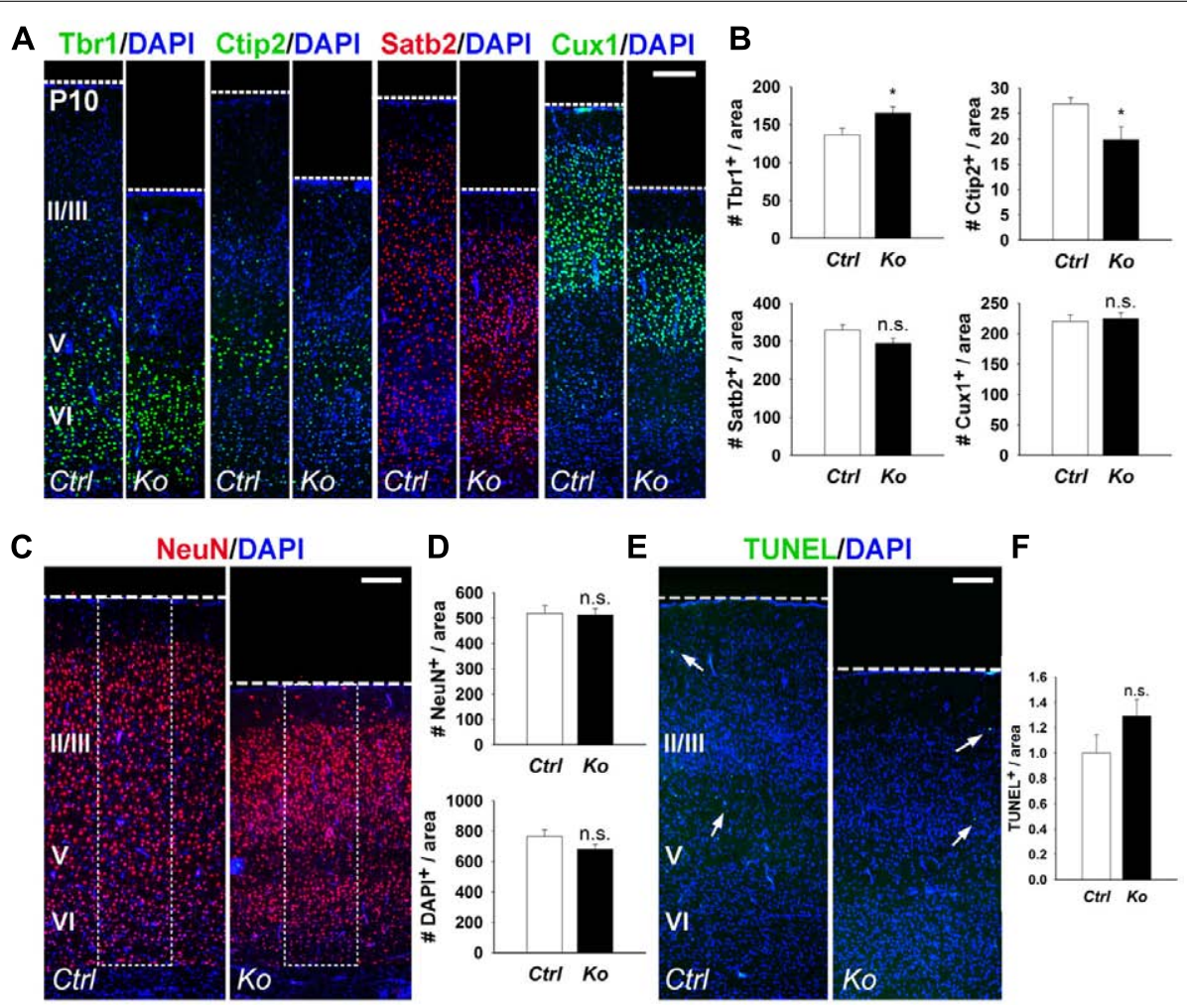

FIGURE 3 | Depletion of miRNA function in postmitotic neurons slightly alters early-born neurons and has no measured effect on neuronal production and cell death at $\mathbf{P 1 0}$. $(\mathbf{A}, \mathbf{B})$ The numbers of early-born neurons labeled with Tbr1 were increased and Ctip2 were decreased in P10 Nex-Cre-Dicer Ko (Ko) cortices, compared to controls (Ctrl). The numbers of late-born neurons labeled with Satb2 and Cux1 were unaffected in P10 Dicer Ko cortices, compared to controls. (C,D) Numbers of $\mathrm{NeuN}^{+}$and $\mathrm{DAPI}^{+}$cells were unaffected in the cortical wall of
P10 Dicer Ko cortices compared to controls. The dashed box indicates the area of quantification. (E) TUNEL assay of coronal cryosections of P10 control and Dicer Ko cortices. Arrows indicate TUNEL ${ }^{+}$cells. (F) Normalized quantification of TUNEL ${ }^{+}$cells per area in the cortical wall of P10 control and Dicer Ko brains. Cortical layers (VI), (V), and (II/III) are labeled. Scale bar: $100 \mu \mathrm{m}$. Data are presented as mean \pm SEM; $n \geq 3$ in all genotypes; $p$ values in relation to control $\left({ }^{*} p<0.04\right)$. n.s., not significant. quantified soma size by measuring the cell body area of Map2and Tuj1-stained neurons. Analysis of soma size revealed that Nex-Cre-Dicer Ko neurons displayed significantly smaller cell body area compared to controls (Figures 6A,B). Our results indicate that miRNA function is required for proper neurite outgrowth and soma size development of differentiated neurons during maturation.

\section{DISCUSSION}

MiRNAs have been found to be crucial for proper development of the CNS. The results reported here underscore the importance of Dicer and miRNAs for neuronal differentiation and maturation. Although removal of Dicer in postmitotic cortical neurons has no immediate impact on neurogenesis, neuronal survival, or layer organization, it has dramatic effects on neurite outgrowth and cortical packing density. Consequently, Dicer-deficient mice exhibited thinner cortical walls and a progressive decline in postnatal growth, resulting in neurodegeneration defects. In conclusion, our results provide evidence that Dicer and miRNAs function is essential for neuronal maturation and that interference with the miRNA pathway results in phenotypes similar to neurodegenerative diseases.
Previous studies have revealed essential roles of miRNAs for neural progenitor proliferation, survival, and differentiation through Dicer ablation during embryonic development of the mouse neocortex (De Pietri Tonelli et al., 2008; Kawase-Koga etal., 2009, 2010; Andersson et al., 2010; Nowakowski et al., 2011). Moreover, limited studies have examined the role of Dicer in specific subpopulations of neurons, such as Purkinje cells, dopaminergic neurons, and excitatory neurons (Kim et al., 2007; Schaefer et al., 2007; Davis et al., 2008). In our mouse model, Dicer is ablated in PNs with the Nex-Cre line. Although the Nex-Cre line displays activity in the cortex by E13.5 (Goebbels et al., 2006), our model system reveals no significant alterations in brain weight or morphology in Dicer deficient mice at P1 (data not shown). This is perhaps caused by a delayed Dicer deletion, which allows a low level of Dicer proteins to continue to process miRNAs and regulate PNs until complete inactivation (Harfe et al., 2005; Kawase-Koga et al., 2009). MiRNAs are expressed in a diverse spectrum and change dynamically during brain development (Lagos-Quintana et al., 2002; Krichevsky et al., 2003; Miska et al., 2004; Sempere et al., 2004; Smirnova et al., 2005). Moreover, conserved complex interactions of multiple genes form a wide regulatory network in the developing cortex (Guillemot, 2005; Molyneaux et al., 2007). 

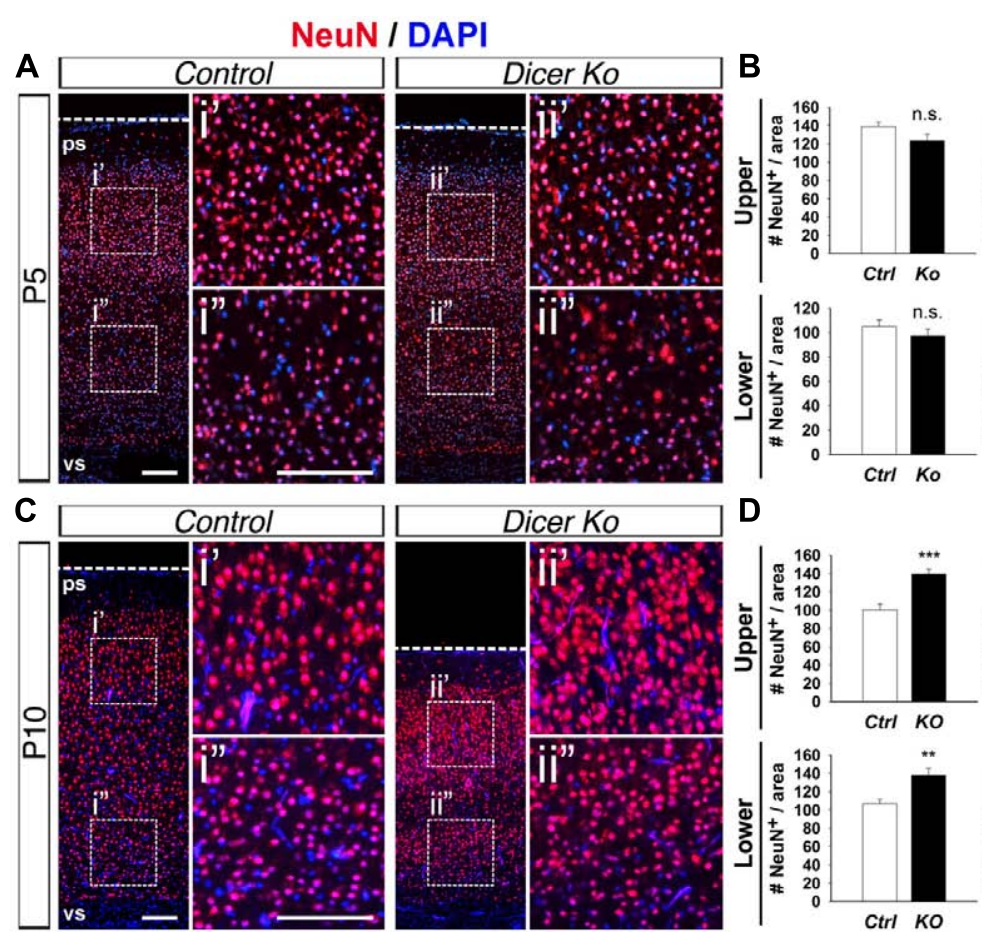

Dicer Ko

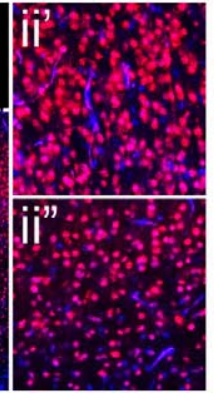

Ctrl Ko

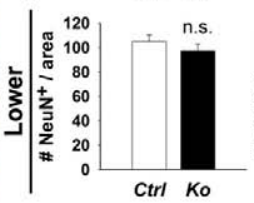

D
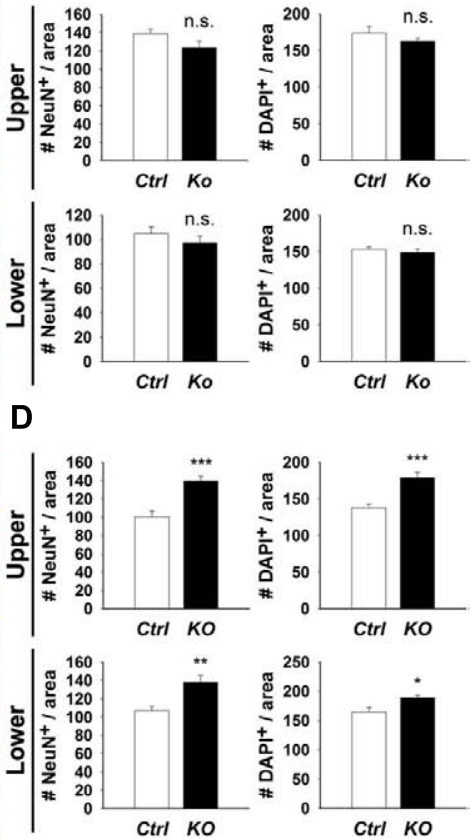

FIGURE 4 | Conditional Dicer ablation increases neuron and cell packing density within the cortex at P10. (A,B) Numbers of $\mathrm{NeuN}^{+}$and $\mathrm{DAPI}^{+}$cells were unaffected in the upper and lower regions of the cortical wall of P5 Nex-Cre-Dicer Ko $(K O)$ cortices compared to controls (Ctrl). (C,D) Numbers of $\mathrm{NeuN}^{+}$and $\mathrm{DAPI}^{+}$cells in the upper and lower regions of the cortical wall were significantly increased in P10 Dicer Ko cortices compared to controls.
The dashed boxes indicate the region shown at higher magnification in panel $i^{\prime}, i^{\prime \prime}, i i^{\prime}, i i^{\prime \prime}$; the boxed area in this region was chosen for subsequent analysis. The ventricular surface (vs) and pial surface (ps) are labeled. Scale bar:

$100 \mu \mathrm{m}$. Data are presented as mean $\pm \mathrm{SEM} ; n \geq 3$ in all genotypes; $p$ values in relation to control $\left({ }^{*} p<0.02,{ }^{* *} p<0.004,{ }^{* *} p<0.0008\right)$. n.s., not significant.

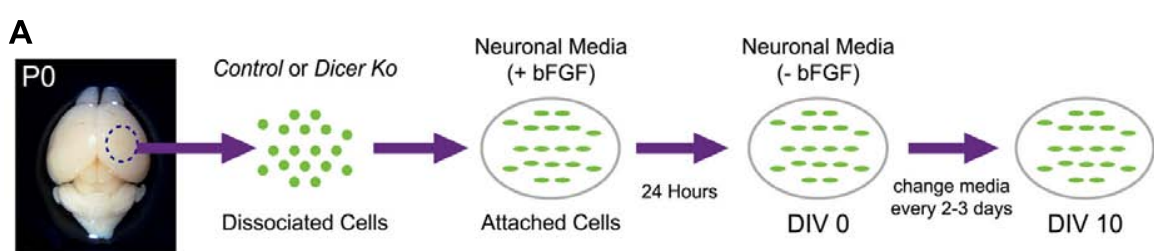

B Map2
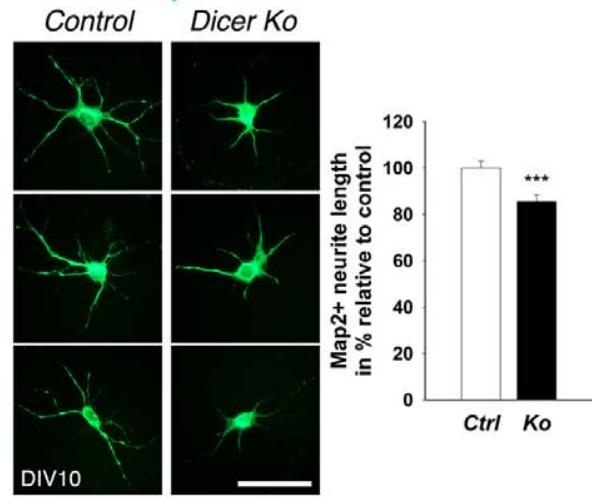

C Tuj1

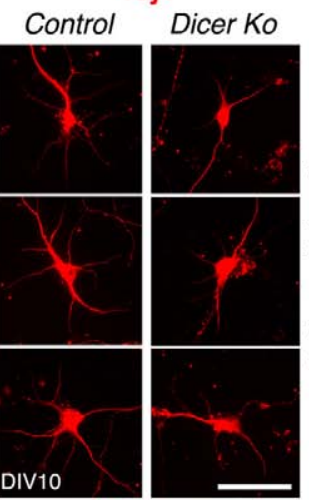

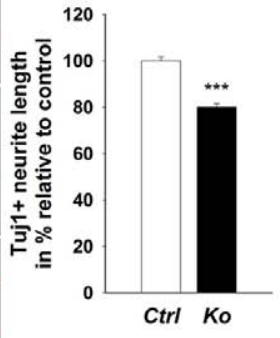

compared to controls $(N=158)$. (C) Measurements of Tuj $1+$ processes displayed shorter neurite outgrowth in DIV 10 Dicer Ko $(N=411)$ neural cultures compared to controls ( $N=423)$. Scale bar: $50 \mu \mathrm{m}$. Data are presented as mean $\pm \mathrm{SEM} ; n \geq 3$ in all genotypes; $p$ values in relation to control (*** $p<0.0008)$. 
A

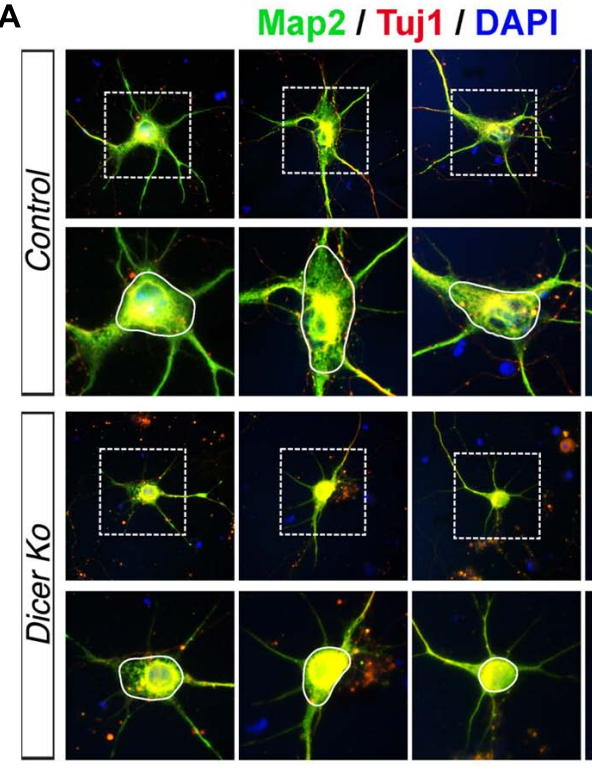

B

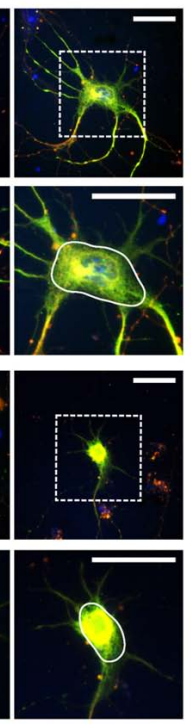

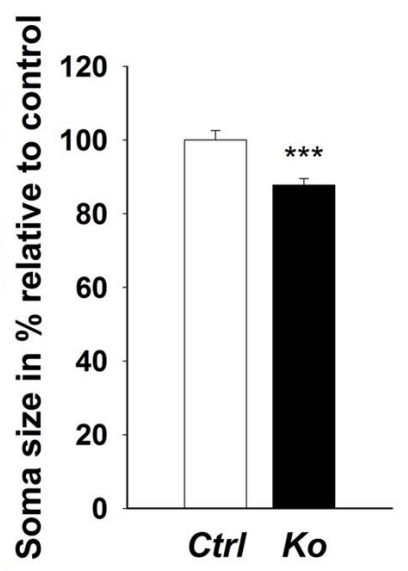

FIGURE 6 | miRNA depletion in maturing cortical neurons causes a reduction of soma size in vitro. (A) Immunofluorescence microscopy of control (Ctrl) and Nex-Cre-Dicer Ko (Ko) primary neural cultures at 10 days in vitro (DIV 10) showing Map2 (green), Tuj1 (red), and DAPI (Jentarra et al., 2010). The dashed box indicates the region shown at higher magnification.
The area encircled by the white line indicates the region of soma size analysis. (B) Measurements of soma size area in DIV 10 primary neurons revealed a significant reduction in Dicer $K_{0}(N=324)$ cell body size compared to controls $(N=259)$. Scale bar: $25 \mu \mathrm{m}$. Data are presented as mean $\pm \mathrm{SEM}$; $n \geq 3$ in all genotypes; $p$ values in relation to control $\left({ }^{* *} p<0.00009\right)$.
As such, the slight alterations in early- and late-born neuron populations in P5 and P10 Nex-Cre-Dicer Ko cortices are perhaps a balanced outcome of a multitude of distinct miRNAs with a variety of regulatory functions and targets.

Given the significant reduction in postnatal cortical growth in Nex-Cre-Dicer Ko brains, it is surprising to find preservation of neuronal cell numbers in the cortex. Moreover, despite a temporal increase of apoptotic cells in P5 cortices, Nex-Cre-Dicer Ko mice do not exhibit significant cell death. These results are in direct contrast to previous studies of Dicer function in Purkinje neurons and DAT-expressing neurons, which found widespread and continuous neurodegeneration and neuronal cell death (Kim et al., 2007; Schaefer et al., 2007). Moreover, compared to Dicer ablation studies in embryonic neural progenitors, which found dramatic apoptotic and differentiation defects, our studies have shown that loss of Dicer activity in postmitotic cortical neurons has minimal impact on neuronal survival (De Pietri Tonelli et al., 2008; Kawase-Koga et al., 2009). This mild apoptosis defect is similar to observations in Dicer Ko mice generated using the CaMKII-Cre line (Davis et al., 2008). These results highlight the diverse and variable functions Dicer and miRNAs carry for cell survival of different cell types at different time points during development.

Although blocking miRNA biogenesis in mature neurons reveals no apparent loss of neurons in the cortex, we have found a major increase in neuronal density in the cerebral cortex. This indicates that neuronal cell volume rather than neuron number is altered by depletion of Dicer and miRNAs in postmitotic cortical neurons. Moreover, direct differentiation of PNs from Dicer deficient cortices in a cell culture system has shown defects in neurite outgrowth (dendrites and axons) and decreased soma size. Decreased neurite outgrowth and increased packing density may contribute to reduced brain size in our Nex-Cre-Dicer Ko mice and in Dicer Ko mice generated using the CaMKII-Cre line (Davis et al., 2008). Moreover, our findings further support previous work, which have found a causal link between specific miRNAs such as miR-134, miR-34, miR-124, miR-9, and miR-132 with neurite outgrowth and elaboration in vitro (Vo et al., 2005; Yu et al., 2008; Agostini etal., 2011; Gaughwin et al., 2011; Clovis et al., 2012; Franke et al., 2012).

In conclusion, our results shed light on the essential role of Dicer-mediated miRNA functions for postmitotic neuronal maturation. Although loss of miRNA function in postmitotic cortical neurons has no definitive impact on neurogenesis, cortical patterning, or cell survival, it causes an atrophic change in neurites (dendrites and axons) and soma size. The aforementioned neurite outgrowth phenotypes are comparable with mouse models of neurodegeneration, which induce generalized atrophy of neuronal soma, dendrites and axons in the brain (Sakai et al., 2006). Increased packing density is also detected in a mouse model of Rett syndrome/X-linked mental retardation (Jentarra et al., 2010). Moreover, abnormally high packing density has been observed in patient brains with Rett syndrome, Williams syndrome, and schizophrenia (Bauman et al., 1995; Selemon et al., 1995; Galaburda et al., 2002). Our model of mature neuron degeneration bears resemblance to cell pathologies associated with schizophrenia and neurodegenerative diseases. As such, understanding the role of specific miRNAs during processes such as neuronal differentiation and maturation may be fundamental to discovering the morphological mechanisms of neurological disorders. 


\section{ACKNOWLEDGMENTS}

We thank members of the Sun laboratory for their valuable discussions. We appreciate $\mathrm{Dr}$ G. Hannon at the Cold Spring Harbor Laboratory and Drs M. Schwab and K. Nave at Max-Planck-Institute of Experimental Medicine

\section{REFERENCES}

Agostini, M., Tucci, P., Steinert, J. R., Shalom-Feuerstein, R., Rouleau, M., Aberdam, D., etal. (2011). microRNA-34a regulates neurite outgrowth, spinal morphology, and function. Proc. Natl. Acad. Sci. U.S.A. 108, 21099-21104. doi: 10.1073/pnas.1112063108

Alcamo, E. A., Chirivella, L., Dautzenberg, M., Dobreva, G., Farinas, I., Grosschedl, R., et al. (2008). Satb2 regulates callosal projection neuron identity in the developing cerebral cortex. Neuron 57, 364-377. doi: 10.1016/j.neuron.2007.12.012

Andersson, T., Rahman, S., Sansom, S. N., Alsio, J. M., Kaneda, M., Smith, J., et al. (2010). Reversible block of mouse neural stem cell differentiation in the absence of dicer and microRNAs. PLoS ONE 5:e13453. doi: 10.1371/journal.pone.0013453

Arlotta, P., Molyneaux, B. J., Chen, J., Inoue, J., Kominami, R., and Macklis, J. D. (2005). Neuronal subtype-specific genes that control corticospinal motor neuron development in vivo. Neuron 45, 207-221. doi: 10.1016/j.neuron.2004.12.036

Bauman, M. L., Kemper, T. L., and Arin, D. M. (1995). Pervasive neuroanatomic abnormalities of the brain in three cases of Rett's syndrome. Neurology 45, 1581-1586. doi: 10.1212/WNL.45.8.1581

Bian, S., and Sun, T. (2011). Functions of noncoding RNAs in neural development and neurological diseases. Mol. Neurobiol. 44, 359-373. doi: 10.1007/s12035-011-8211-3

Britanova, O., de Juan Romero, C., Cheung, A., Kwan, K. Y., Schwark, M., Gyorgy, A., et al. (2008). Satb2 is a postmitotic determinant for upperlayer neuron specification in the neocortex. Neuron 57, 378-392. doi: 10.1016/j.neuron.2007.12.028

Carthew, R. W., and Sontheimer, E. J. (2009). Origins and mechanisms of miRNAs and siRNAs. Cell 136, 642655. doi: 10.1016/j.cell.2009.01.035

Chen, B., Wang, S. S., Hattox, A. M., Rayburn, H., Nelson, S. B., and McConnell, S. K. (2008). The Fezf2-Ctip2 genetic pathway regulates the fate choice of subcortical projection neurons in the developing cerebral cortex. Proc. Natl. Acad. Sci. U.S.A. 105, 11382-11387. doi: 10.1073/pnas.0804918105
Clovis, Y. M., Enard, W., Marinaro, F., Huttner, W. B., and De Pietri Tonelli, D. (2012). Convergent repression of Foxp2 3'UTR by miR-9 and miR132 in embryonic mouse neocortex: implications for radial migration of neurons. Development 139, 33323342. doi: 10.1242/dev.078063

Cubelos, B., Sebastian-Serrano, A., Beccari, L., Calcagnotto, M. E., Cisneros, E., Kim, S., et al. (2010). Cuxl and $\mathrm{Cux} 2$ regulate dendritic branching, spine morphology, and synapses of the upper layer neurons of the cortex. Neuron 66, 523-535. doi: 10.1016/j.neuron.2010.04.038

Davis, T. H., Cuellar, T. L., Koch, S. M., Barker, A. J., Harfe, B. D., McManus, M. T., et al. (2008). Conditional loss of Dicer disrupts cellular and tissue morphogenesis in the cortex and hippocampus. J. Neurosci. 28, 4322-4330. doi: 10.1523/JNEUROSCI.4815-07.2008

De Pietri Tonelli, D., Pulvers, J. N., Haffner, C., Murchison, E. P., HanmiRNAs are essential for survival and differentiation of newborn neurons but not for expansion of neural progenitors during early neurogenesis in the mouse embryonic neocortex. Development 135, 3911-3921. doi: 10.1242/dev.025080

Englund, C., Fink, A., Lau, C., Pham, D., Daza, R. A., Bulfone, A., et al. (2005). Pax6, Tbr2, and Tbr1 are expressed sequentially by radial glia, cells, and postmitotic neurons in developing neocortex. J. Neurosci. 25, 247251. doi: 10.1523/JNEUROSCI.289904.2005

Fineberg, S. K., Kosik, K. S., and Davidson, B. L. (2009). MicroRNAs potentiate neural development. Neuron 64, 303-309. doi: 10.1016/j.neuron.2009.10.020

Frank, C. L., and Tsai, L. H. (2009). Alternative functions of core cell cycle regulators in neuronal migration, neuronal maturation, and synaptic plasticity. Neuron 62, 312-326. doi: 10.1016/j.neuron.2009.03.029

Franke, K., Otto, W., Johannes, S., Baumgart, J., Nitsch, R., and Schumacher, S. (2012). miR124-regulated RhoG reduces neuronal process complexity via ELMO/Dock180/Rac1 and Cdc42 signalling. EMBO J. 31, 2908-2921. doi: 10.1038/emboj.2012.130 non, G. J., and Huttner, W. B. (2008).

for providing mice. This work was supported by the Hirschl/Weill-Caulier Trust (Tao Sun), an NPRP grant (091011-3-260) from the Qatar National Research Fund (Tao Sun) and an R01-MH083680 grant from the NIH/NIMH (Tao Sun).

Galaburda, A. M., Holinger, D. P., Bellugi, U., and Sherman, G. F. (2002). Williams syndrome: neuronal size and neuronal-packing density in primary visual cortex. Arch. Neurol. 59, 1461-1467. doi: 10.1001/archneur.59.9.1461

Gaughwin, P., Ciesla, M., Yang, H., Lim, B., and Brundin, P. (2011). Stage-specific modulation of cortical neuronal development by Mmu-miR-134. Cereb. Cortex 21, 1857-1869. doi: 10.1093/cercor/ bhq262

Goebbels, S., Bormuth, I., Bode, U., Hermanson, O., Schwab, M. H., and Nave, K. A. (2006). Genetic targeting of principal neurons in neocortex and hippocampus of NEXCre mice. Genesis 44, 611-621. doi 10.1002/dvg.20256

Gotz, M., and Huttner, W. B. (2005). The cell biology of neurogenesis. Nat. Rev. Mol. Cell Biol. 6, 777-788. doi: $10.1038 / \mathrm{nrm} 1739$

Guillemot, F. (2005). Cellular and molecular control of neurogenesis in the mammalian telencephalon. Curr. Opin. Cell Biol. 17, 639-647. doi: 10.1016/j.ceb.2005.09.006

Han, W., Kwan, K. Y., Shim, S., Lam, M. M., Shin, Y., Xu, X., et al. (2011). TBR1 directly represses Fezf2 to control the laminar origin and development of the corticospinal tract. Proc. Natl. Acad. Sci. U.S.A. 108, 30413046. doi: 10.1073/pnas.1016723108

Harfe, B. D., McManus, M. T., Mansfield, J. H., Hornstein, E., and Tabin, C. J. (2005). The RNaseIII enzyme Dicer is required for morphogenesis but not patterning of the vertebrate limb. Proc. Natl. Acad. Sci. U.S.A. 102, 10898-10903. doi: 10.1073/pnas.0504834102

Haubensak, W., Attardo, A., Denk, W., and Huttner, W. B. (2004). Neurons arise in the basal neuroepithelium of the early mammalian telencephalon: a major site of neurogenesis. Proc. Natl. Acad. Sci. U.S.A. 101, 31963201. doi: 10.1073/pnas. 0308600100

Hebert, S. S., Papadopoulou, A. S., Smith, P., Galas, M. C., Planel, E., Silahtaroglu, A. N., et al. (2010). Genetic ablation of Dicer in adult forebrain neurons results in abnormal tau hyperphosphorylation and neurodegeneration. Hum. Mol. Genet. 19, 3959-3969. doi: $10.1093 / \mathrm{hmg} / \mathrm{ddq} 311$
Jentarra, G. M., Olfers, S. L., Rice, S. G., Srivastava, N., Homanics, G. E., Blue, M., et al. (2010). Abnormalities of cell packing density and dendritic complexity in the MeCP2 A140V mouse model of Rett syndrome/X-linked mental retardation. BMC Neurosci. 11:19. doi: 10.1186/1471-2202-11-19

Kawase-Koga, Y., Low, R., Otaegi, G., Pollock, A., Deng, H., Eisenhaber, F., et al. (2010). RNAase-III enzyme Dicer maintains signaling pathways for differentiation and survival in mouse cortical neural stem cells. J. Cell Sci. 123, 586-594. doi: 10.1242/jcs.059659

Kawase-Koga, Y., Otaegi, G., and Sun, T. (2009). Different timings of dicer deletion affect neurogenesis and gliogenesis in the developing mouse central nervous system. Dev. Dyn. 238, 2800-2812. doi: 10.1002/dvdy. 22109

Kim, J., Inoue, K., Ishii, J., Vanti, W. B., Voronov, S. V., Murchison, E., et al. (2007). A microRNA feedback circuit in midbrain dopamine neurons. Science 317, 1220-1224. doi: $10.1126 /$ science. 1140481

Kim, V. N., Han, J., and Siomi, M. C. (2009). Biogenesis of small RNAs in animals. Nat. Rev. Mol. Cell Biol. 10, 126-139. doi: 10.1038/nrm2632

Kolk, S. M., Whitman, M. C., Yun, M. E., Shete, P., and Donoghue, M. J. (2006). A unique subpopulation of Tbrl-expressing deep layer neurons in the developing cerebral cortex. Mol. Cell. Neurosci. 32, 200-214. doi: 10.1016/j.mcn.2005.08.022

Kosik, K. S. (2006). The neuronal microRNA system. Nat. Rev. Neurosci. 7, 911-920. doi: 10.1038/nrn2037

Krichevsky, A. M., King, K. S., Donahue, C. P., Khrapko, K., and Kosik, K. S. (2003). A microRNA array reveals extensive regulation of microRNAs during brain development. RNA 9, 1274-1281. doi: 10.1261/rna.5980303

Lagos-Quintana, M., Rauhut, R., Yalcin, A., Meyer, J., Lendeckel, W., and Tuschl, T. (2002). Identification of tissue-specific microRNAs from mouse. Curr. Biol. 12, 735739. doi: 10.1016/S0960-9822(02) 00809-6

Lee, R. C., Feinbaum, R. L., and Ambros, V. (1993). The C. elegans heterochronic gene lin-4 encodes small 
RNAs with antisense complementarity to lin-14. Cell 75, 843-854. doi: 10.1016/0092-8674(93)90529-Y

Merot, Y., Retaux, S., and Heng, J. I. (2009). Molecular mechanisms of projection neuron production and maturation in the developing cerebral cortex. Semin. Cell Dev. Biol. 20, 726-734. doi: 10.1016/j.semcdb.2009.04.003

Miska, E. A., Alvarez-Saavedra, E., Townsend, M., Yoshii, A., Sestan, N., Rakic, P., et al. (2004). Microarray analysis of microRNA expression in the developing mammalian brain. Genome Biol. 5, R68. doi: 10.1186/gb2004-5-9-r68

Molyneaux, B. J., Arlotta, P., Menezes, J. R., and Macklis, J. D. (2007). Neuronal subtype specification in the cerebral cortex. Nat. Rev. Neurosci. 8, 427-437. doi: 10.1038/nrn2151

Murchison, E. P., Partridge, J. F., Tam, O. H., Cheloufi, S., and Hannon, G. J. (2005). Characterization of Dicer-deficient murine embryonic stem cells. Proc. Natl. Acad. Sci. U.S.A. 102, 12135-12140. doi: 10.1073/pnas.0505479102

Noctor, S. C., Flint, A. C., Weissman, T. A., Dammerman, R. S., and Kriegstein, A. R. (2001). Neurons derived from radial glial cells establish radial units in neocortex. Nature 409, 714-720. doi: 10.1038/35055553

Nowakowski, T. J., Mysiak, K. S., Pratt, T., and Price, D. J. (2011). Functional dicer is necessary for appropriate specification of radial glia during early development of mouse telencephalon. PLOS ONE 6:e23013. doi: 10.1371/journal.pone.0023013
Obernosterer, G., Martinez, J., and Alenius, M. (2007). Locked nucleic acid-based in situ detection of microRNAs in mouse tissue sections. Nat. Protoc. 2, 1508-1514. doi: 10.1038/nprot.2007.153

Rakic, P. (2003). Developmental and evolutionary adaptations of cortical radial glia. Cereb. Cortex 13, 541-549. doi: 10.1093/cercor/13. 6.541

Sakai, K., Yamada, M., Sato, T. Tsuji, S., and Takahashi, $H$. (2006). Neuronal atrophy and synaptic alteration in a mouse model of dentatorubral-pallidoluysian atrophy. Brain 129, 2353-2362. doi: 10.1093/brain/awl182

Schaefer, A., O'Carroll, D., Tan, C. L., Hillman, D., Sugimori, M., Llinas, R., et al. (2007). Cerebellar neurodegeneration in the absence of microRNAs. J. Exp. Med. 204, 1553-1558. doi: 10.1084/jem.20070823

Selemon, L. D., Rajkowska, G., and Goldman-Rakic, P. S. (1995). Abnormally high neuronal density in the schizophrenic cortex. A morphometric analysis of prefrontal area 9 and occipital area 17. Arch. Gen. Psychiatry 52: 805-818; discussion 819-820. doi: 10.1001/archpsyc.1995.03950220015005

Sempere, L. F., Freemantle, S., PithaRowe, I., Moss, E., Dmitrovsky, E., and Ambros, V. (2004). Expression profiling of mammalian microRNAs uncovers a subset of brain-expressed microRNAs with possible roles in murine and human neuronal differentiation. Genome Biol. 5, R13. doi: 10.1186/gb-2004-5-3-r13
Shi, Y., Zhao, X., Hsieh, J., Wichterle, H., Impey, S., Banerjee, S., et al. (2010). MicroRNA regulation of neural stem cells and neurogenesis. $J$. Neurosci. 30, 14931-14936. doi: 10.1523/JNEUROSCI.4280-10.2010

Smirnova, L., Grafe, A., Seiler, A., Schumacher, S., Nitsch, R., and Wulczyn, F. G. (2005). Regulation of miRNA expression during neural cell specification. Eur. J. Neurosci. 21, 1469-1477. doi: 10.1111/j.14609568.2005.03978.x

Vo, N., Klein, M. E., Varlamova, O., Keller, D. M., Yamamoto, T., Goodman, R. H., et al. (2005). A cAMPresponse element binding proteininduced microRNA regulates neuronal morphogenesis. Proc. Natl. Acad. Sci. U.S.A. 102, 16426-16431. doi: 10.1073/pnas.0508448102

Volvert, M. L., Rogister, F., Moonen, G., Malgrange, B., and Nguyen, L. (2012). MicroRNAs tune cerebral cortical neurogenesis. Cell Death Differ. 19, 1573-1581. doi: 10.1038/cdd.2012.96

Wightman, B., Ha, I., and Ruvkun, G. (1993). Posttranscriptional regulation of the heterochronic gene lin-14 by lin- 4 mediates temporal pattern formation in C. elegans. Cell 75 , 855-862. doi: 10.1016/00928674(93)90530-4

Yu, J. Y., Chung, K. H., Deo, M., Thompson, R. C., and Turner, D. L. (2008). MicroRNA miR-124 regulates neurite outgrowth during neuronal differentiation. Exp. Cell Res. 314, 2618-2633. doi: 10.1016/j.yexcr.2008.06.002

Yu, J. Y., Wang, T. W., Vojtek, A. B., Parent, J. M., and Turner,
D. L. (2005). Use of short hairpin RNA expression vectors to study mammalian neural development. Methods Enzymol. 392, 186199. doi: 10.1016/S0076-6879(04) 92011-3

Zhang, H., Shykind, B., and Sun, T. (2012). Approaches to manipulating microRNAs in neurogenesis. Front. Neurosci. 6:196. doi: 10.3389/fnins.2012.00196

Conflict of Interest Statement: The authors declare that the research was conducted in the absence of any commercial or financial relationships that could be construed as a potential conflict of interest.

Received: 28 June 2013; accepted: 25 August 2013; published online: 13 September 2013.

Citation: Hong J, Zhang H, Kawase-Koga $Y$ and Sun T (2013) MicroRNA function is required for neurite outgrowth of mature neurons in the mouse postnatal cerebral cortex. Front. Cell. Neurosci. 7:151. doi: 10.3389/fncel.2013.00151

This article was submitted to the journal Frontiers in Cellular Neuroscience. Copyright (c) 2013 Hong, Zhang, KawaseKoga and Sun. This is an open-access article distributed under the terms of the Creative Commons Attribution License (CC BY). The use, distribution or reproduction in other forums is permitted, provided the original author(s) or licensor are credited and that the original publication in this journal is cited, in accordance with accepted academic practice. No use, distribution or reproduction is permitted which does not comply with these terms. 